\title{
Split nitrogen sources effects on nitrogen use efficiency, yield and seed quality of safflower (Carthamus tinctorius L.)
}

\author{
Reza Moradi Talebbeigi, Seyed Abdolreza Kazemeini, Hossein Ghadiri, Mohsen Edalat \\ Department of Crop Production and Plant Breeding, School of Agriculture, Shiraz University, Shiraz, Iran
}

\begin{abstract}
The effects of nitrogen $(\mathrm{N})$ on crop yields have historically been assessed with field trials, but selection and use of the best sources and optimal timing $\mathrm{N}$ applications have a significant role in realizing the maximum potential of oilseeds quality and quantity. This study was conducted to determine the combine effects of $\mathrm{N}$ sources [ammonium nitrate (AN), ammonium sulphate (AS), sulphur coated urea (SCU), and urea (U)] and split $\mathrm{N}$ fertilisation $[(1 / 4,3 / 4,0),(1 / 3,1 / 3,1 / 3),(1 / 2,1 / 2,0)$, and $(1 / 3,2 / 3,0)]$ on safflower (Carthamus tinctorius L.) some growth characters, yield and seed quality, and $\mathrm{N}$ use efficiency based on a split plot design with three replications at the experimental research station, Shiraz University in 2015 and 2016. The highest safflower dry matter $\left(5140.93 \mathrm{~kg} \mathrm{ha}^{-1}\right)$, seed yield $\left(3303.52 \mathrm{~kg} \mathrm{ha}^{-1}\right)$ and protein yield $\left(694.95 \mathrm{~kg} \mathrm{ha}^{-1}\right)$ were achieved with the application of AN fertiliser in a split pattern of $1 / 2,1 / 2,0$ (applying half of the $\mathrm{N}$ at sowing time and the rest at stem elongation), while the highest oil yield $\left(753.09 \mathrm{~kg} \mathrm{ha}^{-1}\right)$ was observed by $\mathrm{U}$ fertiliser and similar split pattern. Applying AN fertiliser and split patterns of $1 / 3,2 / 3,0$ (applying one third of the $\mathrm{N}$ at sowing and two thirds of the $\mathrm{N}$ at stem elongation) and $1 / 4,3 / 4,0$ (applying one quarter of the $\mathrm{N}$ at
\end{abstract}

Correspondence: Seyed Abdolreza Kazemeini, Department of Crop Production and Plant Breeding, School of Agriculture, Shiraz University, Shiraz, Iran.

E-mail: akazemeini@shirazu.ac.ir

Key words: Ammonium nitrate; urea; fertiliser management; oil yield.

Acknowledgements: this project was funded by a grant from the Research Council and Graduate Center of Shiraz University, Shiraz, Iran.

Contributions: the authors contributed equally.

Conflict of interests: the authors declare no potential conflict of interests.

Received for publication: 11 March 2018.

Revision received: 1 June 2018.

Accepted for publication: 12 June 2018.

CCopyright Reza Moradi Talebbeigi et al., 2018

Licensee PAGEPress, Italy

Italian Journal of Agronomy 2018; 13:1246

doi:10.4081/ija.2018.1246

This article is distributed under the terms of the Creative Commons Attribution Noncommercial License (by-nc 4.0) which permits any noncommercial use, distribution, and reproduction in any medium, provided the original author(s) and source are credited. sowing and three quarters at stem elongation) maximised safflower $\mathrm{N}$ uptake efficiency (NUpE) $\left(0.78 \mathrm{~kg} \mathrm{~kg}^{-1}\right)$. However, the highest $\mathrm{N}$ utilisation efficiency (NUtE) (43.70 $\mathrm{kg} \mathrm{kg}^{-1}$ ) was obtained when AN fertiliser in a split pattern of $1 / 2,1 / 2,0$ was applied. On the contrary, applying AS and SCU fertilisers was less effective on safflower performance by all split patterns. It is concluded that applying AN fertiliser in a split pattern of $1 / 3,2 / 3,0$ and or $U$ fertiliser in a split pattern of $1 / 2,1 / 2,0$ not only enhanced safflower growth, yield and seed quality improved, but also increased the $\mathrm{N}$ use efficiency of safflower.

\section{Introduction}

Oilseeds are of great value in nutritional demands of mankind, animal feeding, and medicine. Among them, safflower (Carthamus tinctorius L.) is cultivated for many purposes: its achenes are commonly used as birdseeds and for the extraction of edible or industrial oil; corollas can be used for dyeing fabrics as food colourings and cosmetics and in painting or for producing medicines as pointed out by Danieli et al. (2011) and also as forage crop as reported by Cazzato et al. (2011). An important characteristic of this crop is its adaptation to semi-arid growing conditions owing to a deep root system (2-3 $\mathrm{m}$ in depth) enabling it to obtain moisture from unavailable levels for most crops (Ashkani et al., 2007; Yeilaghi et al., 2012). Quoted by Siadat et al. (2011) and Cazzato et al. (2013), the efficient use of fertilisers is one of the most important factors in maximizing crop yield and sustainability. Rathke et al. (2005) have underlined the significance of higher soil nutrient and particularly $\mathrm{N}$ availability in determining the yield quantity and quality of oilseeds. Moradi-Telavat et al. (2008) reported that nitrogen $(\mathrm{N})$ increased rapeseed yield through increased silique number and seed weight. But a significant negative effect of $\mathrm{N}$ on oil content has been observed (Malhi and Gill, 2004; Siadat et al., 2011). Fismes et al. (2000) and Li et al. (2007) noted that the response of crop to $\mathrm{N}$ fertiliser is influenced by the formulation, fertiliser management, soil properties, and seasonal trends. Muharnmad et al. (2007) reported that the highest seed protein was obtained when rapeseed (Brassica napus L.) treated by calcium ammonium nitrate. Similarly, Ozturk (2010) found that ammonium sulphate (AS) and urea (U) fertilisers gave higher crop yield than ammonium nitrate (AN) fertiliser, while Osman et al. (2014) showed that AN increased growth and yield parameters of rapeseed compared to other $\mathrm{N}$ fertiliser sources.

Because $\mathrm{N}$ availability is an important factor in determining crop productivity, managing fertiliser rate and application timing can be a suitable strategy to improve crop growth and yield when crops need it or when water is available to enhance nutrient uptake (Barlo'g and Grzebisz, 2004; Corbellini et al., 2006). Some studies have shown that, depending on the level of fertility of the initial soil, split applications of $\mathrm{N}$ fertiliser result in higher rates of plant recovery and higher seed yields than under single applica- 
tions (Mossedaq and Smith, 1994; Dordas and Sioulas, 2008; Tedone et al., 2014). Corbellini et al. (2006) and Blandino et al. (2016) reported that the rate and timing of $\mathrm{N}$ applications are crucial factors, not only to obtain high yields, but also to increase protein, gluten and the rheological parameters of wheat. Sheibani and Ghadiri (2012) observed that the application of $U$ at a rate of 304 $\mathrm{kg} \mathrm{ha}^{-1}$, half at the sowing time and the other half at the end of rosette stage of rapeseed, enhanced seed yield up to $35 \%$. Timing of $\mathrm{N}$ fertiliser also increases the efficiency of $\mathrm{N}$ use efficiency, with a higher plant recovery of this mineral and an increase in seed yield and quality, compared to a single application (López-Bellido et al., 2012). Tedone et al. (2014) found that $\mathrm{N}$ application efficiency and $\mathrm{N}$ recovery efficiency in wheat crops increased when $\mathrm{N}$ fertiliser was applied at the stem elongation phase, whereas high amounts of $\mathrm{N}$ at sowing time and tillering, resulted in poor efficiency.

Therefore, it is important to develop strategies to enhance efficiency in uptake and utilisation of mineral nutrients by crops. Although $\mathrm{N}$ fertilisation has been effectively used in agricultural ecosystems' management, the effect of split $\mathrm{N}$ sources on oil crop production is unclear. In order to achieve the maximum potential of the safflower yield quantity and quality, this study was conducted to determine the combined effects of $\mathrm{N}$ sources and splitting $\mathrm{N}$ fertilisation on growth, yield and seed quality of safflower yield, and changes in $\mathrm{N}$ use efficiency.

\section{Materials and methods}

\section{Field experiment}

A 2-year field experiment was conducted at the experimental research station (Badjgah), Shiraz University (52 $46^{\prime}$ E, $29^{\circ} 50^{\prime} \mathrm{N}$ and $1810 \mathrm{~m}$ ), Iran in 2015 and 2016 . The soil was silty clay loam with a $\mathrm{pH}$ of 7.25 and an EC of $0.475 \mathrm{dS} \mathrm{m}^{-1}$. The site was under fallow before cultivation. Total $\mathrm{N}$, mean phosphorus $(\mathrm{P})$ and mean potassium (K) were $0.07 \%, 12 \mathrm{mg} \mathrm{kg}^{-1}$ and $250 \mathrm{mg} \mathrm{kg}^{-1}$, respectively. Monthly average temperature, rainfall, and relative humidity for the last 30 years including the years 2015 and 2016 are shown in Table 1. The experiment was lay-out as split plot based on randomised complete block design with three replications. The treatments consisted of four levels of $\mathrm{N}$ fertiliser sources: $\mathrm{AN}$ $(25 \% \mathrm{~N})$, AS $(21 \% \mathrm{~N})$, sulphur coated urea (SCU; $34 \% \mathrm{~N})$, and U $(46 \% \mathrm{~N})$ as main plot and split patterns of $\mathrm{N}$ were top-dressed (broadcast method) in four levels $[(1 / 4,3 / 4,0),(1 / 3,1 / 3,1 / 3),(1 / 2,1 / 2,0)$, and $(1 / 3,2 / 3,0)]$ as sub plot. Nitrogen fertilisation was carried out at three stages of safflower growing season including sowing time, stem elongation, and flowering (Flemmer et al., 2015). Safflower $\mathrm{N}$ requirement (at a rate of $100 \mathrm{~kg}$ pure $\mathrm{N} \mathrm{ha}^{-1}$ during growing sea- son) was determined according to the soil test analysis. The numbers in each treatment represent the amount of $\mathrm{N}$ fertiliser applied at each stage. For example treatment of $1 / 4,3 / 4,0$ indicated that one quarter of the $\mathrm{N}\left(25 \mathrm{~kg} \mathrm{~N} \mathrm{ha}^{-1}\right)$ at sowing, three quarters $(75 \mathrm{~kg} \mathrm{~N}$ $\mathrm{ha}^{-1}$ ) at stem elongation, and no-N application at flowering stage, respectively. Land preparation practices included plowing, disking and ridging plots (sized 3 by $3 \mathrm{~m}$ ). Each plot was separated by two ridges to avoid cross contamination among plots. The seeds of safflower (Zendehrood cultivar) were sown two $\mathrm{cm}$ deep in rows spaced $15 \mathrm{~cm}$ apart $\left(30\right.$ plants $\left.\mathrm{m}^{-2}\right)$ on March $25^{\text {th }}$ in both years. Triple super phosphate fertiliser was applied at the sowing time at a rate of $50 \mathrm{~kg} \mathrm{ha}^{-1}$ according to the soil test analysis. Other management practices, such as pest control, were conducted according to local agronomic practices unless otherwise indicated. Weeds were controlled by hand hoeing throughout the growing season. The irrigation period was set at 10 day intervals for all treatments. Soil samples were taken at three depths $(30,60$ and $90 \mathrm{~cm})$ at the time of irrigation using the gravimetric method, and moisture content of soil was measured each time before irrigation and each plot was uniformly irrigated by siphon.

\section{Trait measurements}

At the end of the growth period (approximately middle of July in both years), safflower samples were randomly hand harvested from the middle $1 \mathrm{~m}^{2}$ of each plot at maturity and partitioned into stems, leaves, and capitulums. After drying at $76^{\circ} \mathrm{C}$ for $48 \mathrm{~h}$, plant samples were weighed, and dry matter, plant height, capitulum number per $\mathrm{m}^{2}$, number of seeds per capitulum, 1000-seed weight, seed yield, oil yield (Soxhelt method according to Jensen, 2007), protein yield (semi micro-Kjeldahl digestion according to Bremner and Mulvaney, 1982), biological yield, and harvest index:

$$
(H I)=\frac{\text { Seed yield }}{\text { Biological yield }}
$$

were calculated.

Moreover, N uptake efficiency:

$$
(N U p E)=\frac{\text { Total } \mathrm{N} \text { uptake }}{\text { Pure } \mathrm{N} \text { requirment of crop }}
$$

and $\mathrm{N}$ utilisation efficiency:

$$
(N U t E)=\frac{\text { Seed yield }}{\text { Total } \mathrm{N} \text { uptake }}
$$

Table 1. Some monthly weather parameters during the 30-year period from 1986-2016 (the years 2015 and 2016 have been presented separately).

\begin{tabular}{lcccccccccc} 
Months & \multicolumn{3}{c}{ Average temperature $\left({ }^{\circ} \mathrm{C}\right)$} & \multicolumn{3}{c}{ Precipitation $(\mathrm{mm})$} & \multicolumn{3}{c}{ Average relative humidity (\%) } \\
& 2015 & 2016 & 30 -Year & 2015 & 2016 & 30 -Year & 2015 & 2016 & 30 -Year \\
April & 13.90 & 10.20 & 11.23 & 39.50 & 33.50 & 45.82 & 43.10 & 43.16 & 51.85 \\
\hline May & 17.60 & 17.30 & 16.15 & 10.00 & 0.50 & 11.70 & 34.56 & 33.81 & 48.41 \\
June & 23.00 & 20.30 & 20.49 & 0.00 & 0.00 & 0.76 & 24.65 & 27.29 & 39.47 \\
\hline July & 26.00 & 25.29 & 25.43 & 0.00 & 0.00 & 0.30 & 24.48 & 25.31 & 37.49 \\
August & 24.00 & 25.04 & 24.23 & 0.00 & 0.00 & 0.27 & 26.37 & 27.05 & 37.96 \\
\hline Average/Total & 20.90 & 19.62 & 19.33 & 9.90 & 6.80 & 11.77 & 30.63 & 31.32 & 43.04 \\
\hline
\end{tabular}


were estimated according to Rathke et al. (2006). Uptake efficiency is the ability of the plant to remove $\mathrm{N}$ from the soil as nitrate and ammonium ions, while the utilisation efficiency is the ability to use $\mathrm{N}$ to produce seed yield.

\section{Statistical analysis}

Differences between means were tested using SAS 9.1 software (SAS Institute, 2003). Statistical tests included one-way ANOVA (general linear model) followed by least significant difference (LSD) test at 5\% probability level (Petersen, 1994), assuming a normal distribution of the dependent variable data and homogeneity of variances. The effect of year and interaction between year and all treatments were not significant, so the combined data were reported.

\section{Results}

\section{Growth and yield response}

Safflower growth and yield were significantly influenced by split $\mathrm{N}$ fertiliser sources (Table 2). Results showed that the highest plant height $(121 \mathrm{~cm})$ and total dry matter $\left(5140.93 \mathrm{~kg} \mathrm{ha}^{-1}\right)$ were obtained by AN fertiliser and split pattern of $1 / 2,1 / 2,0$. Applying AN fertiliser and split pattern of $1 / 2,1 / 2,0$ increased stem and leaf dry matter approximately 18 and $12 \%$, respectively compared to U fertiliser in a similar split pattern. Likewise, the highest capitulums number (101 per $\mathrm{m}^{2}$ ), seeds number (88), 1000-seed weight (39.25 g) (Table 3), seed yield (3303.52 $\left.\mathrm{kg} \mathrm{ha}^{-1}\right)$, protein yield $(694.95 \mathrm{~kg}$ $\left.\mathrm{ha}^{-1}\right)$, biological yield $\left(8443.60 \mathrm{~kg} \mathrm{ha}^{-1}\right)$, and harvest index (39.14\%) were obtained by AN fertiliser and split pattern of $1 / 2,1 / 2,0$, while the highest oil yield $\left(753.09 \mathrm{~kg} \mathrm{ha}^{-1}\right)$ was obtained when $U$ fertiliser was applied in a split pattern of $1 / 2,1 / 2,0$ (Table 4 ). Applying AN fertiliser and split pattern of $1 / 2,1 / 2,0$ increased seed and protein yields (approximately 22 and 63\%, respectively) compared to $U$ fertiliser in a similar split pattern. On the contrary, applying $U$ fertiliser in a split pattern of $1 / 2,1 / 2,0$ increased oil yield (approximately 5\%) compared to AN fertiliser in a similar split pattern. On the contrary, applying AS and SCU fertilisers increased safflower growth and yield to a lower extent compared to other $\mathrm{N}$ sources (Tables 3 and 4).

\section{Nitrogen use efficiency changes}

The effects of $\mathrm{N}$ sources and split patterns were significant on safflower $\mathrm{N}$ use efficiency (Table 2). AN fertiliser and split patterns of $1 / 4,3 / 4,0$ and $1 / 3,2 / 3,0$ maximised safflower NUpE $(0.78 \mathrm{~kg}$ $\mathrm{kg}^{-1}$ ), however the highest safflower NUtE $\left(43.70 \mathrm{~kg} \mathrm{~kg}^{-1}\right)$ was obtained when AN fertiliser was applied in a split pattern of $1 / 2,1 / 2,0$. In contrast, applying $\mathrm{U}$ fertiliser increased both of them $\mathrm{N}$ efficiencies, when split pattern of $1 / 2,1 / 2,0$ was applied. The lowest $\mathrm{NUpE}\left(0.40 \mathrm{~kg} \mathrm{~kg}^{-1}\right)$ and NUtE $\left(12.31 \mathrm{~kg} \mathrm{~kg}^{-1}\right)$ was observed

Table 2. The summary of the source of variation and the mean square of plant height, total, stem and leaf dry matters, capitulums number, seeds number, 1000-seed weight, seed, oil, protein and biological yields, harvest index, NUpE, and NUtE as affected by split $\mathrm{N}$ fertilisers application.

\begin{tabular}{|c|c|c|c|c|c|c|c|c|}
\hline Source of variation & df & $\begin{array}{l}\text { Plant } \\
\text { height }\end{array}$ & $\begin{array}{l}\text { Total dry } \\
\text { matter }\end{array}$ & $\begin{array}{l}\text { Stem dry } \\
\text { matter }\end{array}$ & $\begin{array}{l}\text { Leaf dry } \\
\text { matter }\end{array}$ & $\begin{array}{l}\text { Capitulums } \\
\text { number }\end{array}$ & $\begin{array}{l}\text { Seeds } \\
\text { number }\end{array}$ & $\begin{array}{c}\text { 1000-seed } \\
\text { weight }\end{array}$ \\
\hline Year & 1 & $176.04^{\mathrm{ns}}$ & $93,543.86^{\mathrm{ns}}$ & $47,530.27^{\mathrm{ns}}$ & $7714.99^{\mathrm{ns}}$ & $13.50^{\mathrm{ns}}$ & $68.34^{\mathrm{ns}}$ & $22.98^{\mathrm{ns}}$ \\
\hline Error (a) & 4 & $107.71^{\text {ns }}$ & $50,675.80^{\text {ns }}$ & $27,490.88^{\text {ns }}$ & $3520.01^{\mathrm{ns}}$ & $3.33^{\text {ns }}$ & $121.34^{\text {ns }}$ & $9.63^{\text {ns }}$ \\
\hline Nitrogen source (N) & 3 & $828.25^{* *}$ & $5,876,466.65^{* *}$ & $4,006,696.02^{* *}$ & $185,611.31^{* *}$ & $1754.34^{* *}$ & $8400.28 * *$ & $531.67^{* *}$ \\
\hline Year * N & 3 & $126.56^{\mathrm{ns}}$ & $48,661.31^{\mathrm{ns}}$ & $25,458.35^{\mathrm{ns}}$ & $3730.41^{\mathrm{ns}}$ & $31.80^{\mathrm{ns}}$ & $26.17^{\mathrm{ns}}$ & $4.85^{\mathrm{ns}}$ \\
\hline Error (b) & 12 & $22.68^{\mathrm{ns}}$ & $31,336.26^{\mathrm{ns}}$ & $16,818.60^{\mathrm{ns}}$ & $2246.66^{\mathrm{ns}}$ & $37.93^{\mathrm{ns}}$ & $175.94^{\mathrm{ns}}$ & $15.82^{\mathrm{ns}}$ \\
\hline Nitrogen split (S) & 3 & $665.36^{* *}$ & $802,840.98^{* *}$ & $433,629.22 * *$ & $56,428.16^{* *}$ & $324.06^{*}$ & $2434.64 * *$ & $73.41^{* *}$ \\
\hline $\mathrm{N} * \mathrm{~S}$ & 9 & $221.13^{* *}$ & $279,727.07 * *$ & $151,058.04 * *$ & $19,721.97 * *$ & $210.41^{*}$ & $261.02 *$ & $24.25 *$ \\
\hline Year*S & 3 & $2.79^{\mathrm{ns}}$ & $1002.38^{\text {ns }}$ & $536.38^{\text {ns }}$ & $72.30^{\mathrm{ns}}$ & $2.41^{\mathrm{ns}}$ & $8.81^{\mathrm{ns}}$ & $2.91^{\text {ns }}$ \\
\hline Year * N*S & 9 & $18.02^{\mathrm{ns}}$ & $336.83^{\text {ns }}$ & $175.93^{\text {ns }}$ & $25.98^{\mathrm{ns}}$ & $5.09^{\mathrm{ns}}$ & $20.94^{\mathrm{ns}}$ & $3.66^{\mathrm{ns}}$ \\
\hline Error $(c)$ & 48 & 54.57 & $58,209.23$ & $30,858.85$ & 4314.73 & 59.82 & 105.18 & 11.04 \\
\hline Source of variation & If & Seed yield & Oil yield & Protein yield & Biological yield & Harvest index & NUpE & NUtE \\
\hline Year & 1 & $129,492.54^{\mathrm{ns}}$ & $17,943.40^{\text {ns }}$ & $6252.06^{\mathrm{ns}}$ & $444,912.1^{\mathrm{ns}}$ & $24.65^{\mathrm{ns}}$ & $0.01^{\text {ns }}$ & $2.71^{\mathrm{ns}}$ \\
\hline Error (a) & 4 & $9639.54^{\mathrm{ns}}$ & $3337.43^{\mathrm{ns}}$ & $156.02^{\text {ns }}$ & $49,970.0^{\text {ns }}$ & $9.69^{\text {ns }}$ & $0.004^{\mathrm{ns}}$ & $21.18^{\text {ns }}$ \\
\hline Nitrogen source (N) & 3 & $18,053,031.56^{* *}$ & $1,339,878.29 * *$ & $696,560.46^{* *}$ & $44,455,591.0^{* *}$ & $1817.71^{* *}$ & $0.43^{* *}$ & $1874.95^{* *}$ \\
\hline Year * N & 3 & $12,808.69^{\mathrm{ns}}$ & $4312.89^{n s}$ & $474.63^{\text {ns }}$ & $97,302.3^{\text {ns }}$ & $2.44^{\mathrm{ns}}$ & $0.0004^{\mathrm{ns}}$ & $11.50^{\mathrm{ns}}$ \\
\hline Error (b) & 12 & $284,342.42^{\mathrm{ns}}$ & $39,855.10^{\mathrm{ns}}$ & $8476.76^{\mathrm{ns}}$ & $326,068.5^{\mathrm{ns}}$ & $39.78^{\mathrm{ns}}$ & $0.005^{\mathrm{ns}}$ & $96.19^{\mathrm{ns}}$ \\
\hline Nitrogen split (S) & 3 & $3,741,045.90^{* *}$ & $139,663.11^{* *}$ & $196,570.70^{* *}$ & $7,382,675.9 * *$ & $370.91^{* *}$ & $0.11^{*}$ & $526.31^{* *}$ \\
\hline$N * S$ & 9 & $708,925.42 * *$ & $39,517.14^{\mathrm{ns}}$ & $57,518.75^{* *}$ & $1,618,282.8^{* *}$ & $44.43^{*}$ & $0.06 *$ & $161.72 *$ \\
\hline Year*S & 3 & $3673.19^{\text {ns }}$ & $588.51^{\mathrm{ns}}$ & $434.90^{\mathrm{ns}}$ & $5840.5^{\mathrm{ns}}$ & $0.46^{\mathrm{ns}}$ & $0.002^{\mathrm{ns}}$ & $6.39^{\mathrm{ns}}$ \\
\hline Year * N*S & 9 & $20,020.43^{\text {ns }}$ & $2262.57^{\mathrm{ns}}$ & $1099.99^{\text {ns }}$ & $18,986.3^{\mathrm{ns}}$ & $2.79^{\mathrm{ns}}$ & $0.002^{\mathrm{ns}}$ & $8.62^{\mathrm{ns}}$ \\
\hline Error (c) & 48 & $173,045.92$ & $14,342.54$ & 3980.01 & $274,979.1$ & 19.60 & 0.005 & 44.33 \\
\hline
\end{tabular}

*Significant at the 0.05 probability level; **Significant at the 0.01 probability level; ${ }^{\text {ns }}$ not significant. 
when AS fertiliser was applied in split patterns of $1 / 3,1 / 3,1 / 3$ and $1 / 2,1 / 2,0$, respectively. Additionally, applying SCU fertiliser and split patterns of $1 / 2,1 / 2,0$ and $1 / 3,2 / 3,0$ enhanced NUpE up to $0.50 \mathrm{~kg}$ $\mathrm{kg}^{-1}$, while NUtE increased up to $21 \mathrm{~kg} \mathrm{~kg}^{-1}$ when SCU fertiliser was applied in a split pattern of $1 / 2,1 / 2,0$ (Table 4 ).

\section{Yield, yield components, and nitrogen efficiency corre- lation}

Correlation results showed that increasing yield components tended to enhance safflower yield and seed yield changes are closely related to the capitulums number $\left(0.86^{* *}\right)$, seeds number

Table 3. Effects of split $\mathrm{N}$ fertilisers application on plant height, total, stem, and leaf dry matters, capitulums number, seeds number, and 1000-seed weight.

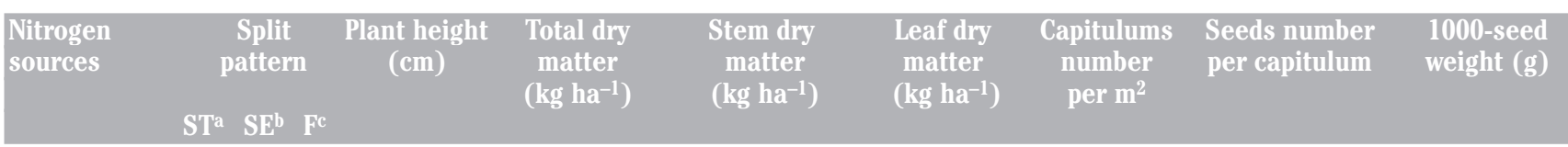

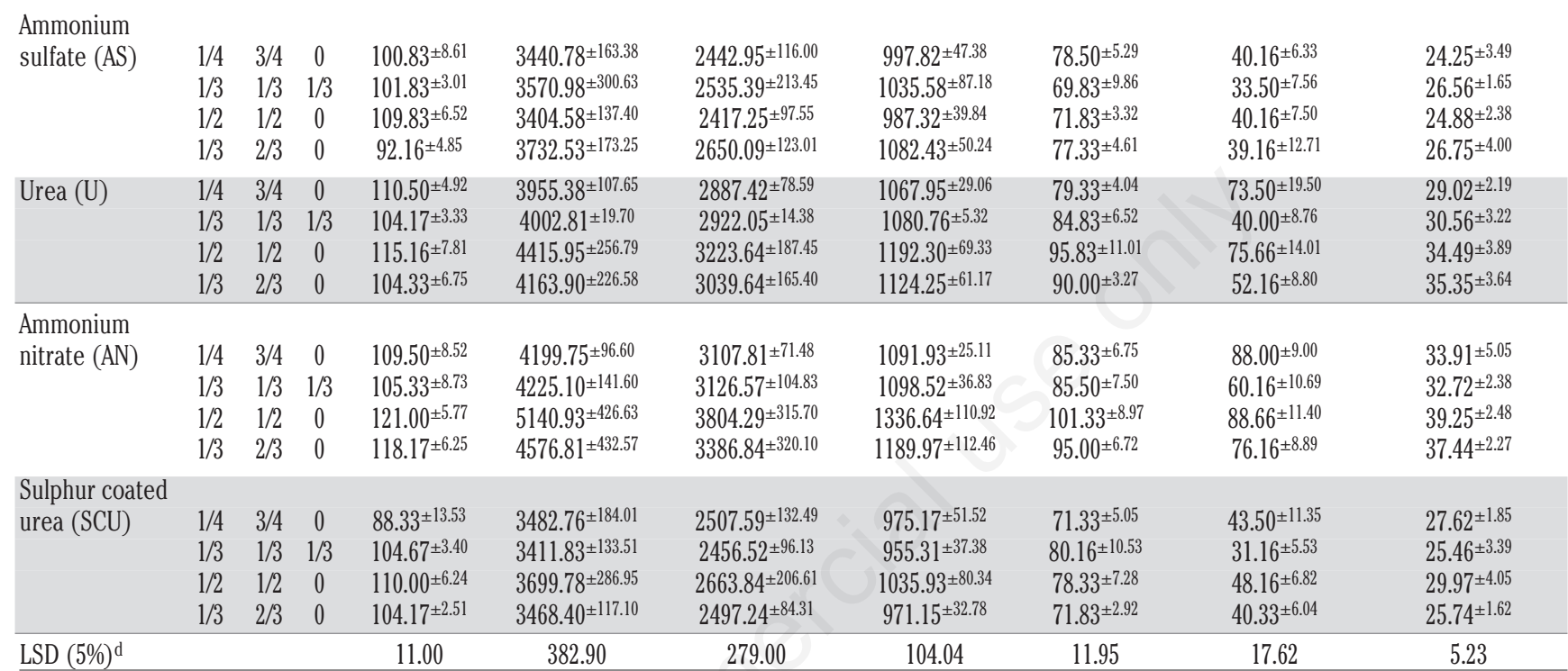

\pm standard deviation with indication of significant differences. a Sowing time; bStem elongation stage; cFlowering stage; deast significant difference at $\mathrm{P}=0.05$.

Table 4. Effects of split $\mathrm{N}$ fertilisers application on seed, oil, protein, and biological yields, harvest index, NUpE, and NUtE.

\begin{tabular}{|c|c|c|c|c|c|c|c|c|c|c|}
\hline $\begin{array}{l}\text { Nitrogen } \\
\text { sources }\end{array}$ & $\mathrm{ST}^{\mathrm{a}}$ & $\begin{array}{l}\text { Split } \\
\text { atte } \\
\text { SEb }^{b}\end{array}$ & & $\begin{array}{l}\text { Seed yield } \\
\left(\mathrm{kg} \mathrm{ha}^{-1}\right)\end{array}$ & $\begin{array}{l}\text { Oil yield } \\
\left(\mathrm{kg} \mathrm{ha}^{-1}\right)\end{array}$ & $\begin{array}{l}\text { Protein yield } \\
\left(\mathrm{kg} \mathrm{ha}^{-1}\right)\end{array}$ & $\begin{array}{l}\text { Biological yield } \\
\left(\mathrm{kg} \mathrm{ha}^{-1}\right)\end{array}$ & $\begin{array}{c}\text { Harvest index } \\
(\%)\end{array}$ & $\begin{array}{c}\text { NUpEe } \\
\left(\mathrm{kg} \mathrm{hat}^{-1}\right)\end{array}$ & $\begin{array}{c}\text { NUtEf } \\
\left(\mathrm{kg} \mathrm{ha}^{-1}\right)\end{array}$ \\
\hline $\begin{array}{l}\text { Ammonium } \\
\text { sulfate (AS) }\end{array}$ & $\begin{array}{l}1 / 4 \\
1 / 3 \\
1 / 2 \\
1 / 3\end{array}$ & $\begin{array}{l}3 / 4 \\
1 / 3 \\
1 / 2 \\
2 / 3\end{array}$ & $\begin{array}{c}0 \\
1 / 3 \\
0 \\
0\end{array}$ & $\begin{array}{c}754.74^{ \pm 59.24} \\
628.43^{ \pm 212.12} \\
713.88^{ \pm 82.52} \\
834.42^{ \pm 354.52}\end{array}$ & $\begin{array}{l}171.25^{ \pm 23.30} \\
143.59^{ \pm 46.93} \\
184.24^{ \pm 13.61} \\
231.92^{ \pm 83.82}\end{array}$ & $\begin{array}{c}80.71^{ \pm 5.48} \\
60.23^{ \pm 20.09} \\
88.09^{ \pm 6.26} \\
81.23^{ \pm 29.67}\end{array}$ & $\begin{array}{c}4195.54^{ \pm 180.97} \\
4199.45^{ \pm 394.78} \\
4118.49^{ \pm 0.10} \\
4566.98^{ \pm 524.91}\end{array}$ & $\begin{array}{l}17.95^{ \pm 1.26} \\
14.82^{ \pm 4.03} \\
17.28^{ \pm 2.19} \\
17.78^{ \pm 5.88}\end{array}$ & $\begin{array}{l}0.48^{ \pm 0.05} \\
0.40^{ \pm 0.01} \\
0.57^{ \pm 0.01} \\
0.51^{ \pm 0.03}\end{array}$ & $\begin{array}{l}15.91^{ \pm 3.21} \\
15.67 \pm 5.60 \\
12.31^{ \pm 1.26} \\
16.00^{ \pm 6.26}\end{array}$ \\
\hline Urea (U) & $\begin{array}{l}1 / 4 \\
1 / 3 \\
1 / 2 \\
1 / 3\end{array}$ & $\begin{array}{l}3 / 4 \\
1 / 3 \\
1 / 2 \\
2 / 3\end{array}$ & $\begin{array}{c}0 \\
1 / 3 \\
0 \\
0\end{array}$ & $\begin{array}{l}1688.06^{ \pm 459.98} \\
1039.35^{ \pm 261.63} \\
2708.77^{ \pm 956.20} \\
1664.46^{ \pm 359.32}\end{array}$ & $\begin{array}{l}485.86^{ \pm 166.65} \\
393.89^{ \pm 181.40} \\
753.09^{ \pm 229.27} \\
512.04^{ \pm 143.29}\end{array}$ & $\begin{array}{c}249.95^{ \pm 65.74} \\
124.86^{ \pm 36.19} \\
425.29^{ \pm 147.61} \\
255.87^{ \pm 61.70}\end{array}$ & $\begin{array}{c}5643.47 \pm 567.91 \\
5042.12^{ \pm 267.46} \\
7124.73^{ \pm 1206.27} \\
5828.38^{ \pm 133.01}\end{array}$ & $\begin{array}{l}29.52^{ \pm 5.26} \\
20.45^{ \pm 4.22} \\
37.19^{ \pm 7.05} \\
28.46^{ \pm 5.53}\end{array}$ & $\begin{array}{l}0.70^{ \pm 0.02} \\
0.65^{ \pm 0.04} \\
0.73^{ \pm 0.13} \\
0.70^{ \pm 0.05}\end{array}$ & $\begin{array}{c}24.07 \pm 6.10 \\
15.80 \pm 3.46 \\
38.99 \pm 18.20 \\
24.07 \pm 7.00\end{array}$ \\
\hline $\begin{array}{l}\text { Ammonium } \\
\text { nitrate (AN) }\end{array}$ & $\begin{array}{l}1 / 4 \\
1 / 3 \\
1 / 2 \\
1 / 3\end{array}$ & $\begin{array}{l}3 / 4 \\
1 / 3 \\
1 / 2 \\
2 / 3\end{array}$ & $\begin{array}{c}0 \\
1 / 3 \\
0 \\
0\end{array}$ & $\begin{array}{l}2599.97^{ \pm 796.02} \\
1685.22^{ \pm 393.04} \\
3303.52^{ \pm 154.97} \\
2715.58^{ \pm 475.33}\end{array}$ & $\begin{array}{c}720.79^{ \pm 270.63} \\
446.03^{ \pm 93.06} \\
715.65^{ \pm 31.93} \\
673.88^{ \pm 188.72}\end{array}$ & $\begin{array}{c}377.51^{ \pm 106.73} \\
186.65^{ \pm 55.21} \\
694.95^{ \pm 125.74} \\
508.24^{ \pm 47.48}\end{array}$ & $\begin{array}{l}6799.73^{ \pm 892.56} \\
5910.33^{ \pm 514.62} \\
8443.60^{ \pm 297.06} \\
7292.34^{ \pm 551.05}\end{array}$ & $\begin{array}{l}37.55^{ \pm 7.11} \\
28.28^{ \pm 4.03} \\
39.14^{ \pm 3.05} \\
37.15^{ \pm 4.94}\end{array}$ & $\begin{array}{c}0.78^{ \pm 0.05} \\
0.67^{ \pm 0.09} \\
0.76^{ \pm 0.05} \\
0.78 \pm 0.08\end{array}$ & $\begin{array}{l}33.01^{ \pm 8.59} \\
24.69^{ \pm 2.18} \\
43.70^{ \pm 4.72} \\
35.25^{ \pm 10.40}\end{array}$ \\
\hline $\begin{array}{l}\text { Sulphur coated } \\
\text { urea (SCU) }\end{array}$ & $\begin{array}{l}1 / 4 \\
1 / 3 \\
1 / 2 \\
1 / 3\end{array}$ & $\begin{array}{l}3 / 4 \\
1 / 3 \\
1 / 2 \\
2 / 3\end{array}$ & $\begin{array}{c}0 \\
1 / 3 \\
0 \\
0\end{array}$ & $\begin{array}{c}840.49 \pm 204.72 \\
640.37 \pm 189.83 \\
1134.83^{ \pm 258.20} \\
743.26^{ \pm 102.44}\end{array}$ & $\begin{array}{l}177.90^{ \pm 28.63} \\
164.46^{ \pm 38.96} \\
238.31^{ \pm 34.22} \\
167.82^{ \pm 42.185}\end{array}$ & $\begin{array}{c}90.21^{ \pm 18.05} \\
76.42^{ \pm 21.00} \\
114.22^{ \pm 25.87} \\
98.98^{ \pm 18.96}\end{array}$ & $\begin{array}{c}4321.90^{ \pm 386.53} \\
4052.13^{ \pm 317.23} \\
4834.63^{ \pm 51.92} \\
4211.70^{ \pm 210.31}\end{array}$ & $\begin{array}{l}19.27 \pm 2.91 \\
15.62 \pm 3.50 \\
23.39 \pm 5.38 \\
17.59 \pm 1.64\end{array}$ & $\begin{array}{l}0.47^{ \pm 0.03} \\
0.44^{ \pm 0.02} \\
0.53^{ \pm 0.02} \\
0.53^{ \pm 0.10}\end{array}$ & $\begin{array}{l}17.42^{ \pm 2.94} \\
14.56^{ \pm 5.00} \\
21.31^{ \pm 4.25} \\
14.10^{ \pm 1.36}\end{array}$ \\
\hline LSD (5\%)d & & & & 706.02 & 221.40 & 111.35 & 865.05 & 7.78 & 0.10 & 10.06 \\
\hline
\end{tabular}

\pm standard deviation with indication of significant differences. ${ }^{2}$ Sowing time; ${ }^{b}$ Stem elongation stage; ${ }^{\circ}$ Flowering stage; ${ }^{2}$ Least significant difference at $\mathrm{P}=0.05$; ${ }^{\mathrm{N}}$ Nitrogen uptake efficiency; ${ }^{\mathrm{f}}$ Nitrogen utilisation efficiency. 
$\left(0.95^{* *}\right)$, and 1000 -seed weight $(0.92 * *)$. Likewise, protein yield increased as $\mathrm{N}$ use efficiency increased especially NUtE $(0.82 * *)$, while increasing $\mathrm{NUpE}$ tended to decrease oil yield $\left(-0.55^{*}\right)$. Additionally, oil yield was negatively correlated with protein yield $\left(-0.65^{*}\right)$, while positive correlation between NUpE and NUtE was observed (Table 5).

\section{Discussion}

It seems that applying $\mathrm{AN}$ and $\mathrm{U}$ fertiliser sources are able to stimulate safflower growth by means of an enlarged leaf canopy and a greater rate of leaf expansion, which increased light interception and enhances photosynthesis (Ozturk, 2010). Additionally, a higher availability of $\mathrm{N}$ in the form of nitrate $\left(\mathrm{NO}_{3}{ }^{-}\right)$and ammonia $\left(\mathrm{NH}_{4}^{+}\right)$increased cations uptake such as potassium $(\mathrm{K})$, calcium (Ca) and magnesium (Mg) (Rathke et al., 2005; Narits, 2010). It seems that increasing safflower growth plays a vital role in enhancing crop yield (Ozturk, 2010). These results are in agreement with those Osman et al. (2014), who reported that in oilseed rape, seed yield is closely related to the capitulums number and the physiological restrictions to capitulum formation are related to poor crop growth and limited leaf expansion. Bagawan and Ravikumar (2001) found a positive correlation between capitulums number and safflower seed yield. Tuncturk and Yildirim (2004) observed that the highest yield response of safflower was obtained by AN and $U$ fertilisers application.

Furthermore, we found that the highest growth and yield responses obtained when safflower was treated by split pattern of $1 / 2$ at sowing time, $1 / 2$ at stem elongation. This high crop growth and performance are the result of high application rate of $\mathrm{N}$ in early growing season as it helps plants to avoid competiting for $\mathrm{N}$ (Ribaudo et al., 2011; Jaynes, 2013). Additionally, Zong et al. (2014) found that the high application rate of $\mathrm{N}$ in mid-growing season resulted in relatively higher plant production compared with the early growing season and this increase was associated with $\mathrm{N}$ enrichment during vegetative-reproductive transition. Narits (2010) observed that the highest oilseed rape growth and yield response was obtained when $\mathrm{N}$ source was split into three period times (sowing time, stem elongation, and flowering stage). Additionally, physiological investigations have shown that the stem elongation stage is important in the $\mathrm{N}$ nutrition of crop when a high N-supply is needed for yield formation (Behrens et al., 2001; Barlo'g and Grzebisz, 2004). Kaefer et al. (2015) reported that oilseed rape growth and yield response were not influenced by $\mathrm{N}$ fertiliser sources, but were significantly influenced by split $\mathrm{N}$ pattern of $1 / 3$ at sowing and $2 / 3$ at top-dressing.

Meanwhile, results showed that adding $\mathrm{AN}$ and $\mathrm{U}$ fertilisers can increase $\mathrm{N}$ use efficiency due to greater access of safflower root to the mass flow of $\mathrm{N}$ especially $\mathrm{NO}_{3}{ }^{-}$(Dawson et al., 2008). $\mathrm{NUpE}$ indicates effectiveness of fertiliser N-recovery due to $\mathrm{N}$ uptake by the plant (Hirel et al., 2007). Furthermore, high NUtE is resulted from effective remobilisation and translocation of $\mathrm{N}$ from vegetative parts (especially stem) of the plant to developing tissues representing strong sinks for $\mathrm{N}$ during the seed-filling period (Masclaux-Daubresse et al., 2010). The positive effect of adding $\mathrm{N}$ sources (especially AN fertiliser) in over production of protein yield and a reduction in oil content is consistent with the findings of Tuncturk and Yildirim (2004) and Farahbakhsh et al. (2006) that increasing $\mathrm{N}$ uptake by plants resulted in production of amino acid and other nitrogenous compounds during peptide bands. When amino acid and other compounds increased, the percentage of fatty acids decreased.

\section{Conclusions}

To enhance the productivity of safflower, different sources and application timing of $\mathrm{N}$ adapted to site conditions are remarkable growing strategies, which could help to realise the maximum potential of safflower quality and quantity. Our results showed that timing of $\mathrm{N}$ fertiliser application has the potential to change safflower agronomical parameters, alter productivity and quality of safflower yield, and influence safflower $\mathrm{N}$ uptake and utilisation efficiency. Overall, our findings suggested that applying AN fertiliser in a split pattern of $1 / 3,2 / 3,0$ and /or $U$ fertiliser in a split pattern of $1 / 2,1 / 2,0$ not only improves safflower growth, yield and seed quality, but also enhances the $\mathrm{N}$ use efficiency of safflower.

Table 5. Correlation between yield, yield components, and $\mathrm{N}$ efficiency of safflower.

Seed yield No. Capitulums No. Seeds 1000-seed weight Oil yield Protein yield Harvest index NUpE NUtE

\begin{tabular}{|c|c|c|c|c|c|c|c|c|}
\hline Seed yield & 1.00 & & & & & & & \\
\hline No. Capitulums & $0.86^{* *}$ & 1.00 & & & & & & \\
\hline No. Seeds & $0.95^{* *}$ & $0.72^{* *}$ & 1.00 & & & & & \\
\hline 1000-seed weight & $0.92 * *$ & $0.86^{* *}$ & $0.82 * *$ & 1.00 & & & & \\
\hline Oil yield & $0.64^{*}$ & $0.48^{*}$ & $0.54^{*}$ & $0.44^{*}$ & 1.00 & & & \\
\hline Protein yield & $0.86^{* *}$ & $0.74^{* *}$ & $0.79 * *$ & $0.69^{*}$ & $-0.65^{*}$ & 1.00 & & \\
\hline Harvest index & $0.98^{* *}$ & $0.84^{* *}$ & $0.97^{* *}$ & $0.91^{* *}$ & $0.58^{*}$ & $0.81^{* *}$ & 1.00 & \\
\hline NUpE & $0.89 * *$ & $0.80^{* *}$ & $0.88^{* *}$ & $0.85^{* *}$ & $-0.55^{*}$ & $0.79^{* *}$ & $0.92^{* *}$ & 1.00 \\
\hline NUtE & $0.98 * *$ & $0.86^{* *}$ & $0.92 * *$ & $0.90 * *$ & $-0.65^{*}$ & $0.82^{* *}$ & $0.96 * *$ & $0.81^{* *}$ \\
\hline
\end{tabular}

*Significant at the 0.05 probability level; ** Significant at the 0.01 probability level. 


\section{References}

Ashkani J, Pakniyat H, Emam Y, Assad MT, Bahrani MJ, 2007. The Evaluation and Relationships of Some Physiological Traits in Spring Safflower (Carthamus tinctorius L.) under Stress and Non-Stress Water Regimes. J. Agr. Sci. Tech. 9:267-77.

Bagawan I, Ravikumar RL, 2001. Strong undesirable linkages between seed yield and oil components- A problem in safflower improvement. pp 103-107 in Proc. $5^{\text {th }}$ Int. Safflower Conf. USA.

Barlo'g P, Grsebisz W, 2004. Effect of timing and nitrogen fertilizer application on winter oilseed rape (Brassica napus L.). I. growth dynamics and seed yield. J. Agron. Crop Sci. 190:305-13.

Behrens T, Horst WJ, Wiesler F, 2001. Effect of rate, timing and form of nitrogen application on yield formation and nitrogen balance in oilseed rape production. In: W.J. Horst (Eds.), Plant Nutrition: Food Security and Sustainability of AgroEcosystems through Basic and Applied Research. Kluwer Academic Publishers, Dordrecht, pp 800-1.

Blandino M, Marinaccio F, Reyneri A, 2016. Effect of late-season nitrogen fertilization on grain yield and flour rheological quality and stability in common wheat, under different production situations. Ital. J. Agron. 11:107-13.

Bremner JM, Mulvaney CS, 1982. Total nitrogen. In: Page AL (Eds.), Methods of Soil Analysis. Part 2. $2^{\text {nd }}$ Ed. American Society of Agronomy, Madison (WI), USA.

Cazzato E, Laudadio V, Corleto A, Tufarelli V, 2011. Effects of harvest date, wilting and inoculation on yield and forage quality of ensiling safflower (Carthamus tinctorius L.) biomass. J. Sci. Food Agric. 91:2298-302.

Cazzato E, Tufarelli V, Laudadio V, Stellacci AM, Selvaggi M, Leoni B, Troccoli C, 2013. Forage yield and quality of emmer (Triticum dicoccum Schübler) and spelt (Triticum spelta L.) as affected by harvest period and nitrogen fertilization. Acta Agr. Scand. B Soil Plant Sci. 63:571-8.

Corbellini M, Perenzin M, Boggini G, Bellocchi G, Scudellari D, Monotti M, 2006. Balance sheet method assessment for nitrogen fertilization in bread wheat: I. Yield and quality. Ital. J. Agron. 3:331-41.

Danieli PP, Primi R, Ronchi B, Ruggeri R, Rossini F, Del-Puglia S, Cereti CF, 2011. The potential role of spineless safflower (Carthamus tinctorius L.) as fodder crop in central Italy. Ital. J. Agron. 6:19-22.

Dawson JC, Huggins DR, Jones SS, 2008. Characterizing nitrogen use efficiency in natural and agricultural ecosystems to improve the performance of cereal crops in low-input and organic agricultural systems. Field Crops Res. 107:89-101.

Dordas CA, Sioulas C, 2008. Safflower yield, chlorophyll content, photosynthesis, and water use efficiency response to nitrogen fertilization under rain-fed conditions. Ind. Crops Prod. 27:75-85.

Farahbakhsh H, Pakgohar N, Karimi A, 2006. Effect of nitrogen and sulphur fertilizers on yield, yield components and oil content of oilseed rape (Brassica napus L.). Asian J. Plant Sci. 5:112-5.

Fismes J, Vong PC, Guckert A, Frossard E, 2000. Influence of sulphur on apparent N-use efficiency, yield and quality of oilseed rape (Brassica napus L.) grown on a calcareous soil. Eur. J. Agron. 12:127-41.

Flemmer AC, Franchini MC, Lindstrom LI, 2015. Description of safflower (Carthamus tinctorius) phonological growth stages according to the extended BBCH scale. Ann. Appl. Biol. 166:331-9.

Hirel B, Le Gouis J, Ney B, Gallais A, 2007. The challenge of improving nitrogen use efficiency in crop plants: towards a more central role for genetic variability and quantitative genetics within integrated approaches. J. Exp. Bot. 58:2369-87.

Jaynes DB, 2013. Nitrate loss in subsurface drainage and corn yield as affected by timing of side-dress nitrogen. Agr. Water Manage. 130:52-60.

Jensen WB, 2007. The origin of the Soxhelt extractor. J. Chem. Educ. 84:1913-4.

Kaefer JE, Richart A, Nozaki MH, Daga J, Campagnolo R, Follmann PE, 2015. Canola Response to Nitrogen Sources and Split Application. Rev. Bras. Eng. Agríc. 19:1042-8.

Li X, Hu C, Delgado JA, Zhang Y, Ouyand Z, 2007. Increase nitrogen use efficiency as a key mitigation alternative to reduce nitrate leaching in north china plain. Agr. Water Manage. 89:137-47.

López-Bellido L, Munoz-Romero V, Benítez-Vegaa J, FernándezGarcía P, Redondo R, López-Bellido RJ, 2012. Wheat response to nitrogen splitting applied to a Vertisols in different tillage systems and crop-ping rotations under typical Mediterranean climatic conditions. Eur. J. Agron. 43:24-32.

Malhi SS, Gill KS, 2004. Placement, rate and source of N, Seed row opener and seedling depth effects on canola production. Can. J. Plant Sci. 84:719-9.

Masclaux-Daubresse C, Daniel-Vedele F, Dechorgnat J, Chardon F, Gaufichon L, Suzuki A, 2010. Nitrogen uptake, assimilation and remobilization in plants: challenges for sustainable and productive agriculture. Ann. Bot. 105:1141-57.

Moradi-Telavat MR, Siadat SA, Nadian H, Fathi G, 2008. Effect of nitrogen and boron on canola yield and yield components in Ahwaz, Iran. Int. J. Agric. Res. 3:415-22.

Mossedaq F, Smith DH, 1994. Timing nitrogen application to enhance spring-wheat yields in a Mediterranean climate. Agron. J. 86:221-226.

Muharnmad N, Cheerna MA, Wahid MA, Ahmad N, Zaman M, 2007. Effect of Source and Method of Nitrogen Fertilizer Application on Seed Yield and Quality of Canola (Brassica Napus L). Pak. J. Agr. Sci. 44:74-8.

Narits L, 2010. Effect of nitrogen rate and application time to yield and quality of winter oilseed rape (Brassica napus L. var. Oleifera subvar. biennis). Agron. Res. 20:671-86.

Osman EAM, El-Galad MA, Khatab KA, Zahran FAF, 2014. Canola productivity as affected by nitrogen fertilizer sources and rates grown in calcareous soil irrigated with saline water. Glob. J. Sci. Res. 2:137-43.

Ozturk O, 2010. Effects of source and rate of nitrogen fertilizer on yield, yield components and quality of winter rapeseed (Brassica napus L.). Chil. J. Agr. Res. 70:132-41.

Petersen RG, 1994. Agricultural Field Experiments: Design and Analysis. CRC Press.

Rathke GW, Christen O, Diepenbrock W, 2005. Effects of nitrogen source and rate on productivity and quality of winter oilseed rape (Brassica napus L.) grown in different crop rotations. Field Crops Res. 94:103-13.

Rathke GW, Behrens T, Diepenbrock W, 2006. Integrated Nitrogen Management Strategies to Improve Seed Yield, Oil Content and Nitrogen Efficiency of Winter Oilseed rape (Brassica napus L.): A Review. Agr. Ecosyst. Environ. 117:80-108.

Ribaudo M, Delgado J, Hansen L, Livingston M, Mosheim R, Williamson J, 2011. Nitrogen in Agricultural Systems: Implications for Conservation Policy. U.S. Department of Agriculture, Economic Research Service, Washington, DC. ERR-127.

SAS Institute, 2003. SAS user's guide. SAS Institute, Cary (NC), USA. 
Sheibani S, Ghadiri H, 2012. Effect of split nitrogen fertilization and herbicide application on soil weed seed bank in wheat (Triticum aestivum L.) and oilseed rape (Brassica napus L.) rotation. J. Biol. Environ. Sci. 6:25-33.

Siadat SA, Moradi-Telavat MR, Fathi GH, Mazarei M, Alamisaeid KH, Mousavi SH, 2011. Rapeseed (Brassica napus L.) response to nitrogen fertilizer following different previous crops. Ital. J. Agron. 6:199-203.

Tedone L, Verdini L, Grassano N, Tarraf W, De-Mastro G, 2014. Optimizing nitrogen in order to improve the efficiency, ecophysiology, yield and quality on one cultivar of durum wheat. Ital. J. Agron. 9:49-54.
Tuncturk M, Yildirim B, 2004. Effects of different forms and doses of nitrogen fertilizers on safflower (Carthamus tinctorius L.). Pak. J. Biol. Sci. 7:1385-9.

Yeilaghi H, Arzani A, Ghaderian M, Fotovat R, Feizi M, Pourdad SS, 2012. Effect of Salinity on Seed Oil Content and Fatty Acid Composition of Safflower (Carthamus tinctorius L.) Genotypes. Food Chem. 130:618-25.

Zong N, Song M, Shi P, Jiang J, Zhang X, Shen Z, 2014. Timing patterns of nitrogen application alter plant production and $\mathrm{CO}_{2}$ efflux in an alpine meadow on the Tibetan Plateau, China. Pedobiologia. 57:263-9. 